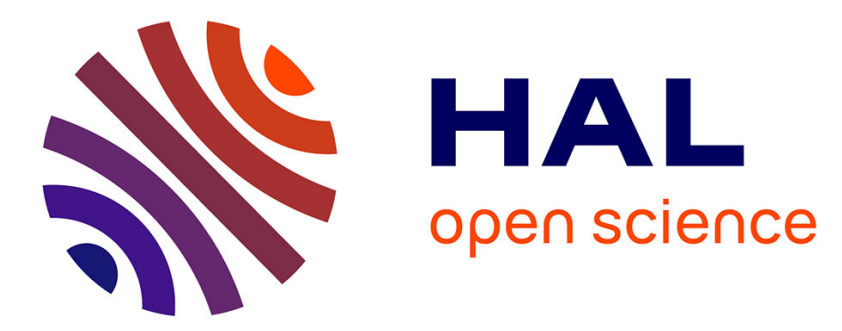

\title{
Simulation of the Emotion Dynamics in a Group of Agents in an Evacuation Situation
}

van Minh Le, Carole Adam, Richard Canal, Benoit Gaudou, Tuong Vinh Ho, Patrick Taillandier

\section{- To cite this version:}

van Minh Le, Carole Adam, Richard Canal, Benoit Gaudou, Tuong Vinh Ho, et al.. Simulation of the Emotion Dynamics in a Group of Agents in an Evacuation Situation. Pacific Rim workshop on Agent-based modeling and simulation of Complex Systems, 2010, Kolkata, India. pp.604-619, 10.1007/978-3-642-25920-3_44. hal-00692147

\section{HAL Id: hal-00692147 \\ https://hal.science/hal-00692147}

Submitted on 29 Apr 2012

HAL is a multi-disciplinary open access archive for the deposit and dissemination of scientific research documents, whether they are published or not. The documents may come from teaching and research institutions in France or abroad, or from public or private research centers.
L'archive ouverte pluridisciplinaire HAL, est destinée au dépôt et à la diffusion de documents scientifiques de niveau recherche, publiés ou non, émanant des établissements d'enseignement et de recherche français ou étrangers, des laboratoires publics ou privés. 


\title{
Simulation of the Emotion Dynamics in a Group of Agents in an Evacuation Situation
}

\author{
Le Van Minh ${ }^{1,2}$, Carole Adam ${ }^{3}$, Richard Canal ${ }^{1,4}$, Benoit Gaudou ${ }^{5,6}$, \\ Ho Tuong Vinh ${ }^{1,4}$, and Patrick Taillandier ${ }^{6}$ \\ 1 Institut de la Francophonie pour l'Informatique (IFI), Hanoi, Vietnam \\ 2 Danang University (UDN), Danang, Vietnam \\ 3 RMIT University, Melbourne, Australia \\ 4 UMI 209 UMMISCO, Institut de Recherche pour le Développement (IRD), \\ Bondy, France \\ ${ }^{5}$ Université de Toulouse, Toulouse, France \\ ${ }^{6}$ Institut de Recherche en Informatique de Toulouse (IRIT), UMR CNRS 5505, \\ Toulouse, France \\ \{minh.levan246, carole.adam.rmit, benoit.gaudou, ho.tuong.vinh, \\ patrick.taillandier\}@gmail.com, richard.canal@auf.org
}

\begin{abstract}
Nowadays, more and more emergency evacuation simulations are used to evaluate the safety level of a building during an emergency evacuation after an accident. The heart of this kind of simulations is the simulation of human behavior because simulation results depend for a big part on how this behavior is simulated. However, human behaviors in a real emergency situation are determined by a lot of cognitive mechanisms. In order to make the simulation more realistic, plenty of factors (e.g. innate characteristics, perception of the environment, internal rules, personality and even emotions) that affect human behaviors must be taken into account. This paper focuses on the influence of emotions, and more precisely on the influence of their dynamics and propagation from an agent to another. The main contribution of this work is the development of a model of emotions taking into account their dynamics and their propagation and its integration in an evacuation simulation. The first results of the simulation show the benefits of considering emotion propagation.
\end{abstract}

Keywords: emotion, emotional agent, emotion propagation, emergency evacuation.

\section{Introduction}

Nowadays, the computing power available on any personal computer allows one to create simulations including thousands of agents, as for example in simulations of the traffic in a city, or simulations of the emergency evacuation from a building... In order to make the simulation as realistic as possible, each agent in the simulation is designed to operate autonomously (which means that the 
agent is not controlled by any central agent and he makes his decisions using his own cognitive resources) and to interact with other agents.

Many researches show that adding emotions (e.g. joy, fear, anger, hope...) into these agents provides huge benefits because emotions improve the quality of agents' behaviors and then the quality of the whole simulation [13. Indeed emotions play a very important role in human beings' life by influencing their decision-making and reasoning processes and their interactions with others 68 . Therefore, we should not ignore emotions when creating a virtual human simulation.

The recent studies in the domain of artificial agents allow one to give each agent ways to express pertinent emotions [7] and to reason about not only his own emotions but also about others agents' ones [1. Critical issues remain concerning the "transmission" of emotions among agents in the simulation, the emergence of a common emotion in a group of agents and the effect of this emotion on each individual.

We thus propose in this paper a model of emotions taking into account both the emotion dynamics (when emotions appear and how their intensity level evolves over time) and the emotion propagation (how they are "sent" and "received" and how a received emotion influences the receiver). In this paper we focus on fear (with various intensity levels) and test our model by implementing it into agents used in a simulation of emergency evacuation in a burning shopping center.

This paper is organized as follows. Section 2 presents a brief state of the art on emotions. In Section 3, we present our model of emotions, emotion dynamics and propagation. Furthermore, in Section 4, we present the implementation of our model and discuss in Section 5 the first results of our simulation.

\section{State of the Art}

\subsection{Simulation of Pedestrian Evacuation}

Plenty of simulations of emergency evacuation have been developed [182019. Most of them concentrate on human behaviors, and more particularly on their movement, in an emergency situation. The typical model of this kind of simulations is the pedestrian evacuation model developed by Crooks on the RepastJ platform 14]. In this simulation, agents representing human beings leave their office when the fire appears. This simulation is focused on the human movement and on the role of obstacles on the passage to the door: e.g. the wall of the room, the table...

Since the human behaviors in the real world are much more complex than those of the agents in the simulation (e.g. planned tasks of a person are often updated in case of a stressful situation), many researchers have proposed to introduce additional factors to make the agents' behavior more human-like.

${ }^{1}$ Interested readers can download the model on the website http://www.casa.ucl.ac.uk/andrew/repastmodels/fire/fire.zip 
In particular, Musse and Thalmann described a hierarchical model of virtual crowds for real-time simulations [12. In their simulation, the human behaviors executed by simulated agents are classified in three types: innate or scripted behaviors, behaviors defined by rules and behaviors controlled by external factors. Furthermore, Hollmann et al. [9] improved the human behaviors model in urgent situations by adding the two following attributes: available time and estimated required time. When these two variables reach a particular threshold, the agent changes his behavior (e.g. he accelerates in order to get to the goal location, changes the goal location or even ignores some of the planned tasks to concentrate on the ultimate goal).

As mentioned previously, emotions play an important role in human behaviors in particular in emergency situations. We choose to integrate emotions in evacuation simulations. Therefore the first question is how to define and describe emotions.

\subsection{Theories of Emotions}

In the 19th century, James and Lange proposed a physiological theory of emotion [10. Their theory indicates that the human autonomous nervous system creates physiological events like heart rate and respiration in response to various human experiences. The emotions are the sentiments that appear as results of this physiological change. According to this theory, humans can express their emotions via their physiological states and can guess the emotions of others based on this kind of expression.

While this physiological theory advocates that emotions result from physiological factors, many scientists defend the idea that cognitive factors are very important in the process of emotion triggering. They propose various so called cognitive theories. In the domain of cognitive theories of emotions, Arnold [3] and Lazarus 11 argue that human beings always evaluate what they perceive and the emotion triggered by this perception is thus the result of an appraisal process. They proposed the cognitive appraisal theory. Following these purely psychological researches, Ortony, Clore and Collins [15] define a typology of emotions (known as the OCC typology) depending on the type of stimulus appraised and various appraisal variables. They distinguish three kinds of stimuli: events, actions of agents, aspects of objects. The 20 emotions defined are deeply related to mental attitudes, this eases their integration into artificial agents.

In [5], Cabanac proposed a four-dimension model of consciousness. In this model, every state of consciousness is described in 4 dimensions: the qualitative dimension, the intensive dimension, the hedonic dimension and the time dimension. He then proposed a definition of emotions based on his model of consciousness: "Emotion is any mental experience with high intensity and high hedonicity" [5].

In 4], Bosse et al. proposed a model of emotion contagion. In their model, the propagation of emotions depends on six factors: the current level of the sender's emotion, the current level of the receiver's emotion, the extent to which the sender expresses the emotion, the receiver's openness or sensitivity for emotions, 
the strength of the channel from the sender to the receiver and the tendency to adapt emotions upward or downward [4].

\subsection{Existing Models of Emotional Agent}

In [16, Parunak et al. propose a model of emotions for situated agents called DETT. "DETT (Disposition, Emotion, Trigger, Tendency) is an environmentally mediated model of emotion that captures the essential features of the widelyused OCC (Ortony, Clore, Collins) model of emotion" [16]. Contrarily to most agents architectures using the OCC typology, in DETT emotions are triggered by the perception module (rather than being the result of an internal reasoning).

In [22], Zoumpoulaki et al. propose a multi-agent framework for emergency evacuation. The multi-agent model presented in the paper is a combination of the BDI (Belief, Desire and Intention) architecture [17] for the agent's reasoning process, the OCEAN (Openness, Conscientiousness, Extraversion, Agreeableness and Neuroticism) for the model of personality and the OCC model for emotions. This kind of framework can be used to simulate emotions in controllable situations, but in the case of an extremely serious emergency, when the situation becomes truly chaotic, we argue that people do not have enough time to make reasoned decisions but rather make decisions based on simple heuristics such as emotions. In the sequel we consider situations of extreme emergency; that is why we have only simple agents feeling extreme emotions (like fear). Their behavior is mainly directed by the emotion they are feeling and the emotions received from other agents.

\section{Proposed Model of Emotional Agent}

\subsection{A Two-Dimensional Model of Emotion for Emergency Evacuation Simulations}

As described in the previous section, we consider an emotion as a particular mental state which is triggered by the individual appraisal of various stimuli. Stimuli in our case will be either events of the environment or behaviors of other agents, both being perceived by the agent. The subject appraises these stimuli according to his knowledge and his current emotions.

From the psychological four-dimensional model proposed by Cabanac [5] and the multi-agent framework integrating personality and emotion proposed by Zoumpoulaki et al. 22, it appears that the more emotions are simulated, the more realistic the simulation becomes but the more complicated relevant results will be extracted from the simulation, which leads to difficulties in the evaluation phase when we evaluate the role of emotions and of their propagation in emergency situations. In order to emphasize the effect of emotions and of their transmission, we propose a simple model of emotion - a two-dimension model of emotions - which allows us to have simulation results that remain simple to analyze. Our model is the reduction of Cabanac's four-dimensional model [5] by 


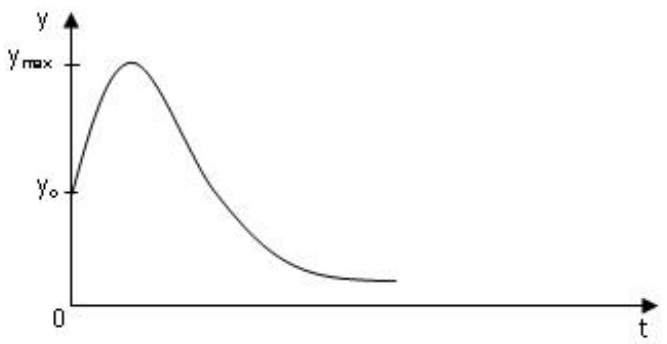

Fig. 1. Decay of the emotion

ignoring the qualitative dimension (Axis $\mathrm{x}$ ) and the hedonic dimension (Axis $\mathrm{z}$ ). We consider that the emotion triggered in every emergency evacuation is the fear. We concentrate on this emotion and the two remaining dimensions represent its intensity and the duration of the emotion. Thus, the emotional model proposed is a two-dimensional model with 2 axes: the emotion intensity (Axis y) and the emotion duration (Axis t).

"Axis y" represents the intensity of the emotion. In our proposition, the value of $y(t)$ is continuous and always positive. If $y(t)<\epsilon$ (a very small threshold), the emotion disappears. The initial value of $y(t)$ (i.e. when the emotion is triggered) is calculated by the appraising process. We suppose that an agent has the capability to calm down over time and thus reduce the intensity of his emotion. Then the value of $y(t)$ decreases gradually following the formula $y(t)=y(t-1) / \alpha,(\alpha>1)$

(1) (with $\alpha$ is the decay coefficient).

"Axis t" represents the duration of the emotion. Due to the decay equation (1), the intensity of the emotion will follow the evolution presented in Figure 1. in the ideal case where there is no other stimulus.

\subsection{Architecture of the Emotional Agent}

We present in Figure 2 the architecture of our emotional agent.

Simplifications. In the sequel, we consider the three following simplifications. They allow us to simplify conceptually and computationally our model and thus improve the simulation performance and make the simulation more effective.

Simplification 1. The current emotion of the agent does not have any influence on the process of perception. The current emotion is taken into account only in the process of evaluation of the perception outputs (emotional appraisal) that triggers emotion.

Simplification 2. There is only one emotion in the agent at one moment. This also means that only one emotion can be taken into account in the appraisal process and that this process can create only one emotion. 


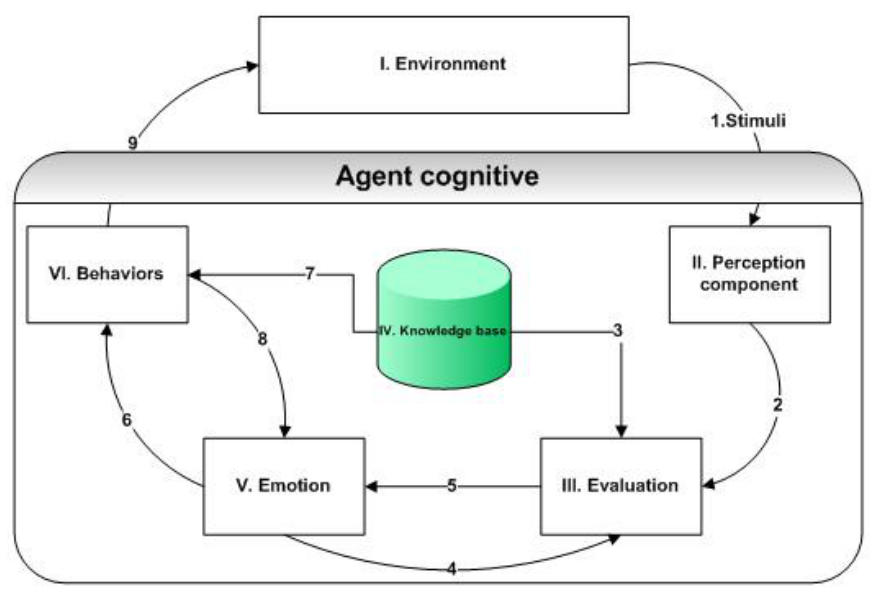

Fig. 2. Model of the emotional agent

Simplification 3. The agent acts immediately once the emotion appears. The agent does not update his knowledge base before performing an action.

Description of the architecture. We describe here the main process of the agent architecture.

1. Perception of the stimuli (Perception component in the Figure 2): first, the agent perceives the stimulus (coming from Arrow 1). In this work, the type of emotion simulated is the fear. Therefore, only the stimuli which contribute to fear are perceived, the rest is ignored. According to the source of the stimuli, the agent classifies the stimulus into 1 of 2 possible types.

a. Indirect stimulus: This type of stimulus is caused by the environment. They do not trigger directly an emotion. They can only affect the intensity of existing emotions. For example: a child who is alone in the middle of the graveyard, under the heavy rain; if this child is scared, any additional noise will increase the level of his fear.

b. Direct stimulus: This type of stimulus is caused by events of the environment or by the behavior of other agents. Each stimulus provokes one type of emotion with a specific intensity.

2. Appraisal of the stimuli (Evaluation component in Figure 2): The results of the perception (Arrow 2) are appraised according to the knowledge of the agent (Arrow 3) and his current emotion (Arrow 4). There are 2 types of stimulus so there are also 2 types of evaluation. In the end of this process, the agent creates an emotion.

a. Evaluation of indirect stimuli: the agent searches in his knowledge base for rules relating to the indirect stimuli perceived. If some rules are found, the agent acquires the global variable $\left(\gamma_{i}\right)$. As the stimulus can have either a positive effect or a negative effect on the agent, this global variable $\left(\gamma_{i}\right)$ can have a positive value or a negative value. With one stimulus, $\gamma_{i}$ can be different from an agent to 


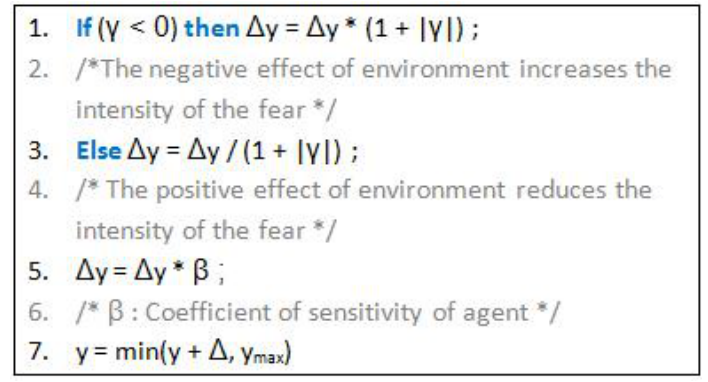

Fig. 3. Algorithm to calculate the intensity of the fear

another one according to his knowledge base: an agent can give a positive value to $\gamma_{i}$, whereas another agent can give it a negative value. For example, consider a child in a dark room; the darkness is the indirect stimulus; if this child can normally see, this stimulus is negative; if this child is blind, darkness does not scare him; so this stimulus will not be perceived or will have a neutral value. After having evaluated all the indirect stimuli, the agent has a set of $\gamma_{i}$ that he aggregates with: $\gamma=\sum_{i>0} \gamma_{i}$.

b. Evaluation of direct stimuli: the agent finds in his knowledge base the rules matching with the direct stimuli perceived. According to rules found, the agent acquires an additional intensity value $(\Delta y)$.

c. Computation of the intensity: the intensity of the temporary emotion is computed by the algorithm presented in the Figure 3 .

In the algorithm, " $y$ " represents the intensity of the fear. When " $y$ " reaches a determined threshold, the agent will change his behavior. The maximal value of " $y$ " is " $y_{\max }$ " which is defined as an input parameter of the simulation that limits an overgrowing emotion intensity. In our model, we consider that agents perceive other agents' emotions depending on their sensitivity factor $\alpha$. This sensitivity coefficient is a positive constant and varies from an agent to another one. According to this coefficient, an agent can perceive emotion more easily than others.

3. Computation of behaviors (Behaviors component in Figure 2): once the new emotion has been created, the agent reacts immediately to the stimulus from the environment. Initially, the agent finds in his knowledge base the rules related to the emotion and then executes the corresponding behavior. The agent executes two kinds of action in one step: he reduces the intensity according to the formula (1) and reacts to the stimulus. When the agent reacts to the stimulus, his behavior affects not only the environment but also the other agents. The other agents perceive thus these behaviors and interpret them as stimuli.

\subsection{Model of Emotion Propagation}

According to the agent model proposed, an agent appraises the stimuli caused by the actions of other agents in the process of evaluation. When an agent reacts 


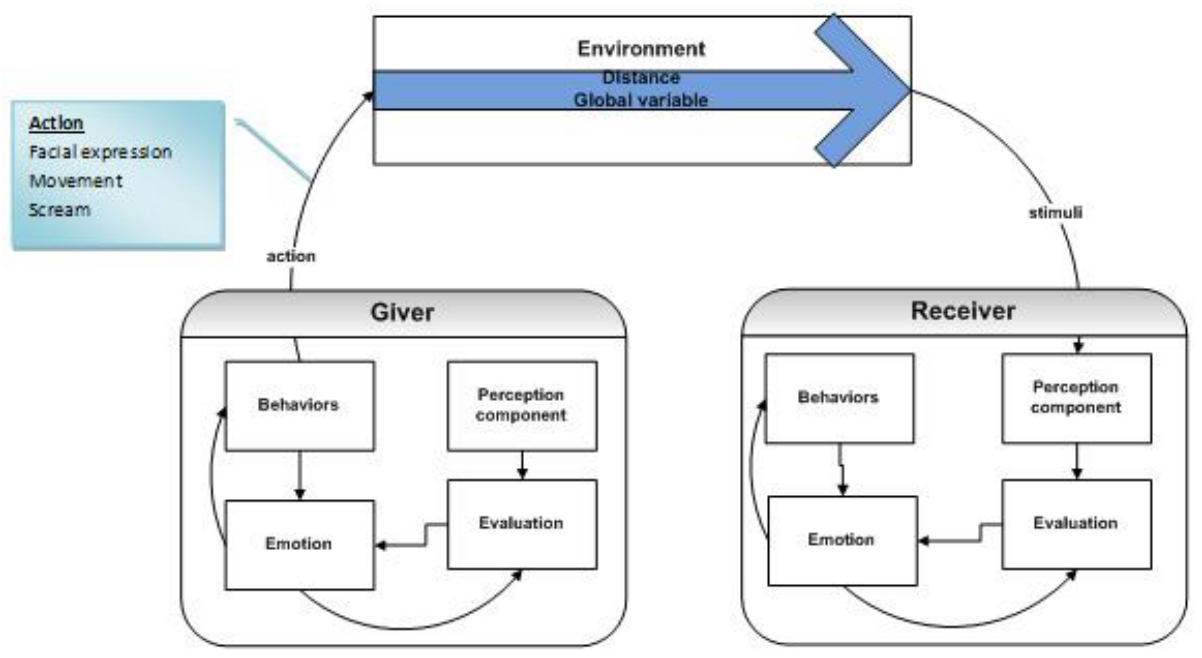

Fig. 4. Emotional propagation model

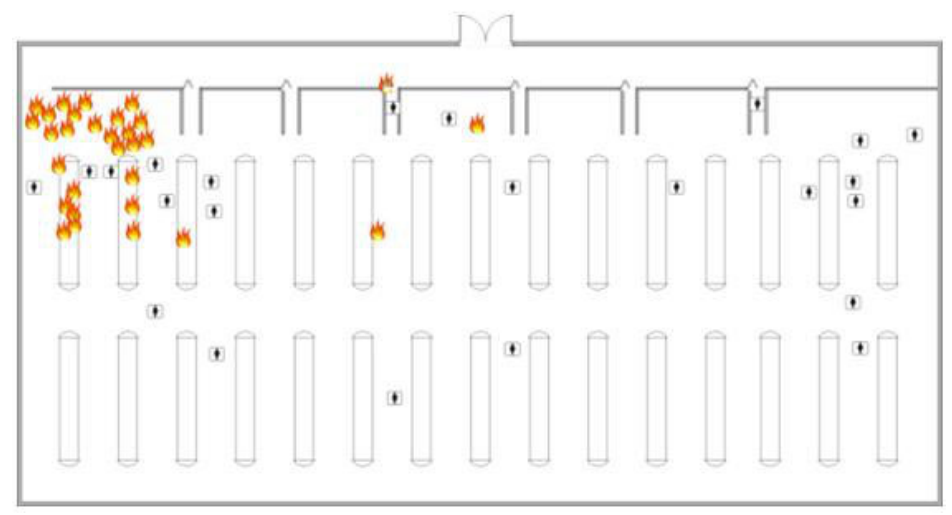

Fig. 5. Overview of the simulation

to the environment, he expresses his emotion via his behavior. Then, an agent can recognize the emotion of the other ones according to the behaviors that he perceives. The emotion of an agent can thus spread over a group of agents. Figure 4 describes the way an agent gives his emotion to others via his behaviors.

\section{Implementation of the Emotional Agent}

In this section we present the situation that we want to simulate, the details of the agents involved in this simulation and how we can improve their behavior by introducing emotions. The model is implemented on the GAMA platform [2 21]. 


\subsection{Description of the Application Case: Pedestrian Evacuation in a Burning Shopping Center}

The simulation aims at representing the scenario described below. In a shopping center, while people are shopping, the fire appears. Figure 5 presents the overview of the simulation. The people who see the fire may be scared. They may scream or change their movement speed. Other agents who perceive these actions may feel their fear. With this simulation we aim at highlighting the important role of emotions (in this case, the fear) in an emergency evacuation, and in particular the fact that the casualty rate can highly be increased, in the case of a panicked crowd.

\subsection{Description of the Agents}

In our simulation, we use two kinds of agent: agents representing human beings and agents representing fire. In the sequel we describe in details the implementation of both kinds of agent.

\section{Human Agent}

\begin{tabular}{|l|l|}
\hline Attributes & Methods \\
\hline \hline Size: agent size & Wander(speed:float): \\
Perception_range: radius within & the agent moves randomly \\
which an agent can perceive a stimulus & GoToTarget(speed:float): \\
Propagation_range: radius within & the agent goes to his target \\
which an agent can propagate & AvoidObstacle(): \\
information to others & The agent avoids the obstacles \\
Emotion_type: type of emotion (in & and the other agents \\
this model, emotion_type is "fear") & PerceiveStimuli(): \\
Emotion_intensity: intensity of & the agent perceives stimuli within \\
the emotion & his range of perception \\
Sensitivity_ability ( $\alpha):$ the sensitivity & PropagateInformation(): \\
of an agent. The greater this attribute & the agent propagates information \\
is, the more sensible the agent is & within his range of propagation \\
Decay_ability: the capability to reduce & Reflex(): the agent evaluates the \\
the intensity of emotion. The greater & stimulus in order to choose his \\
this coefficient is, the faster the & behavior \\
emotion disappears & ChangeColor(color:Color): \\
Global_variable: the global effect of & the agent changes his color \\
environment on each agent. This & \\
coefficient is set randomly at the & \\
beginning of the simulation & \\
\hline
\end{tabular}




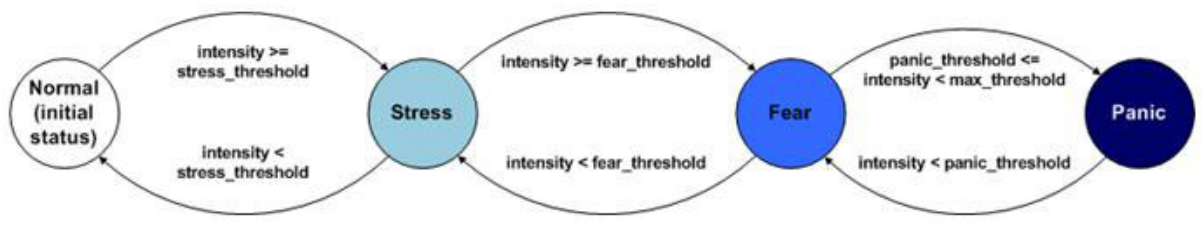

Fig. 6. Levels of the fear

\begin{tabular}{|r|l|l|l|l|l|}
\hline No & Behaviors & Normal & Stress & Fear & Panic \\
\hline 1 & Display in color & & & & \\
\hline 2 & Avoid obstacle & yes & yes & yes & yes \\
\hline 3 & Perceive stimuli & yes & yes & yes & yes \\
\hline 4 & Wander with normal speed & yes & no & no & no \\
\hline 5 & Quit the shopping center with normal speed & no & yes & no & no \\
\hline 6 & Quit the shopping center with great speed & no & no & yes & no \\
\hline 7 & Wander with great speed & no & no & no & yes \\
\hline 8 & Propagate information to others & no & yes & yes & yes \\
\hline
\end{tabular}

Fig. 7. List of behaviors

Fire agent

\begin{tabular}{|l|l|}
\hline Attributes & Methods \\
\hline \hline Size: size of one piece of fire & Propagate(): the agent propagates \\
Duration: duration of the fire & himself within the range of propagation \\
Propagation_range: range within & \\
which the fire can spread at each step & \\
\hline
\end{tabular}

\subsection{Description of the Emotion (The Fear)}

As mentioned above, the emotion that influences the most the behavior of agents in an emergency situation is the fear. We choose thus to limit the emotions involved in the simulation to the fear. In our simulation, we distinguish four levels of fear (depending on the intensity level of the emotion): normal, stress, fear and panic. Figure 6 presents these levels.

At each level of fear, the agent may execute different behaviors or a behavior with different manners (e.g. wander with a normal speed when the agent is in the state normal or wander with a high speed when he is panicked). In our model we proposed seven behaviors described in Figure 7.

1. Display in color: the agent uses this behavior to show graphically his current fear level. At each level of fear the agent uses the method ChangeColor(color:Color) to show a particular color (white, light blue, blue, dark blue). 
2. Avoid obstacle: this is a fundamental behavior. At every step an agent moves, he invokes the method AvoidObstacle() to avoid collisions with obstacles (wall, other agents...)

3. Perceive the stimuli: this is also a fundamental behavior: this method is invoked at each step. The agent can perceive the fire or get emotional information from the behavior of other agents.

4. Wander with normal speed: this behavior shows that the agent is in a nonstressful state. The agent wanders with a normal speed if he is not scared. In this case, the agent invokes the method Wander(speed : float) with the normal value of speed.

5. Quit the shopping center with normal speed: this behavior shows that the agent is aware of a fire. The agent who is scared with the average intensity keeps his reasoning capabilities: he can thus find a way to escape. The agent invokes the method GoToTarget(speed : float) with the normal value of speed.

6. Quit the shopping center with great speed: this behavior is similar to the behavior 5 but in this case, the agent is truly afraid: the value of the speed is thus higher.

7. Wander with great speed: similar to the behavior 4 but in this case the speed is higher. This behavior illustrates the case when the fear is so intense that the agent becomes panicked, loses awareness and thus cannot control himself.

8. Propagate the information: As soon as the agent feels any kind of fear (stress, fear or panic), he invokes the method PropagateInformation() to spread this emotional information to other agents.

\section{Experimentation}

In this section, we present the tests led on the emergency evacuation model. In order to evaluate the role of the emotional factor in the emergency evacuation, we propose two measures that we use to monitor our model: Emotional Rate (ER) is the percentage of people who are in a fear state in a unit of time; Survivor Rate (SR) is the percentage of people who succeed to escape the building. Our experimentation is separated into three models: a model without emotion, a model with emotion but without propagation and a model with emotion and propagation. In each test case, the parameters of the emotional factors are set with the average value so that the simulation is closer to actual situations.

\subsection{Model without Emotion}

In this test case, we set the values of the propagation_range and of the perception_range to zero, which means that the human agent cannot perceive the emotion of other agents. In this case, the human agents are not aware of the danger. And when the fire propagates in a wide area, these agents do not change their behavior to survive. In consequence, the survivor rate (SR) is very low. 


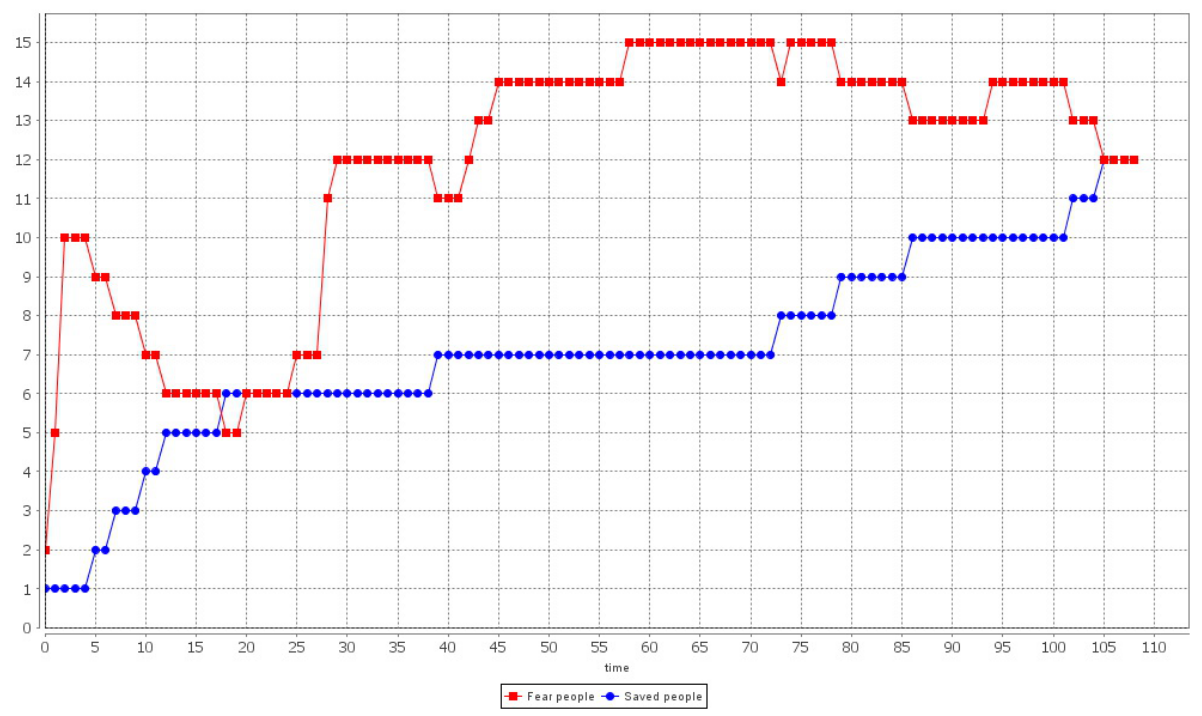

Fig. 8. Evolution of the emotion rate and of the number of survivors

\subsection{Model with Emotions but without Propagation}

In this test case, the value of the propagation_range is zero and the perception_range is set randomly in its definition interval. In this case, the human agents can themselves perceive the fire which can trigger fear but cannot propagate their emotion to others. Only the people who see the fire may become afraid and then flee the danger to save their life. The others who do not see the fire are not scared. This is why the emotional rate (ER) is higher than in the first test case but still low. With the people who are not aware of the danger, it may be too late to survive because the fire spreads quickly and blocks the exit. Thus, in this test case, the survivor rate (SR) is still low. The chart in Figure 8 shows the progress of the two indicators during the simulation.

\subsection{Model with Emotions and Propagation}

In this test case, the value of the propagation_range and of the perception_range are set randomly in their definition interval, which means that the people can feel the fear by perceiving the stimuli from the environment and can inform the others about their fear. They can perceive not only the fire but also the behaviors of others. In the chart in the Figure 9, at step 40, the emotional propagation happens, the emotional rate (ER) jumps thus significantly and then the survivor rate (SR) increases quickly. That is why the survivor rate is higher.

\subsection{Discussion}

After having executed the simulation 100 times and making statistics on the data obtained, we find that the benefits of the emotional factors in the simulation of 


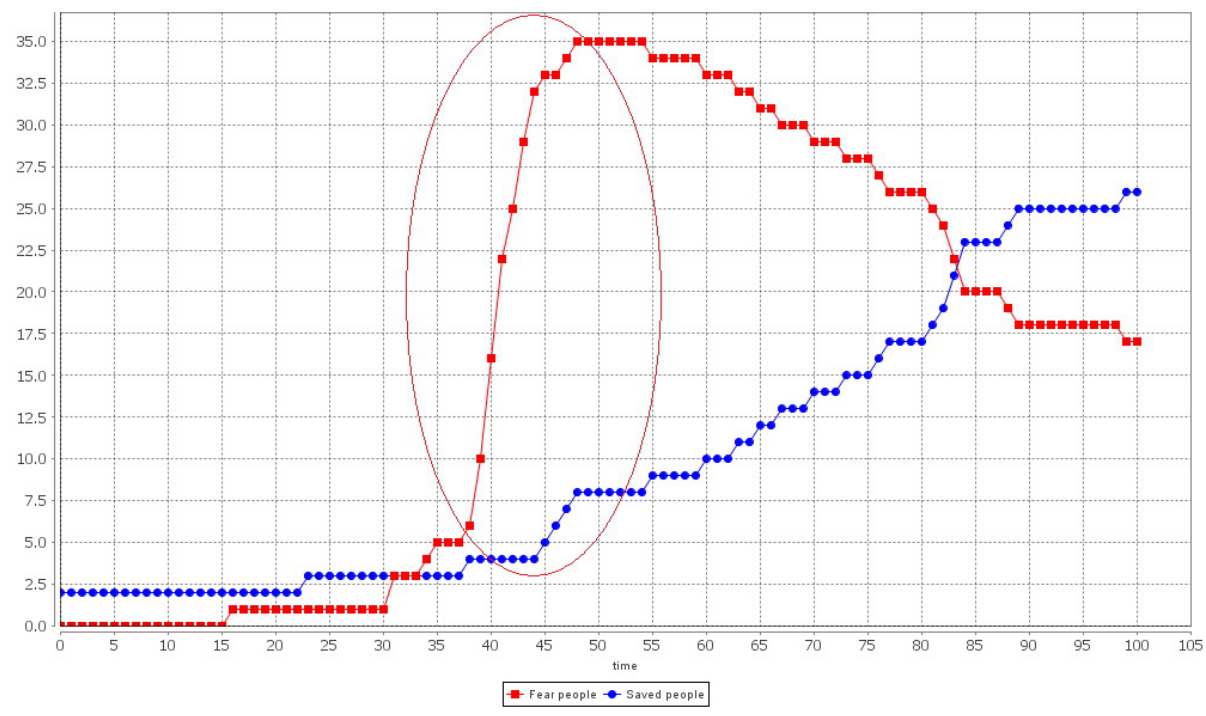

Fig. 9. Evolutions of the emotion rate and the number of survivors

the emergency evacuation is very important. In the chart of the Figure 10, in the case of emotions with propagation, the percentage of survivors is the highest $(45.03 \%)$.

We can summarize our model by responding to following questions.

How an emotion appears? The agent perceives stimuli; he evaluates them according to his knowledge and his current emotion; then, a new emotion appears.

Which may influence the appraisal process of the stimuli? The stimuli, the empathy of the agent and effects of the environment influence the evaluation of emotion. The intensity of emotion of each agent is different to each other, although they perceive the same stimuli at the same time. When the agents perceive the fire, some may be scared, others may not. This is all because of the empathy.

How can an agent show his emotion? Through his behaviors, an agent shows his current emotion. In our simulation, these behaviors are: appearance change (like the human facial expression), the movement speed or other ways to inform other agents (scream, cry...).

How can the propagation of emotions happen? An agent perceives the others' behaviors, evaluates them like any environmental stimuli. Then, an emotion appears. Thus, the emotion spreads over agents.

In our research, we found that there is an amplification of the emotional intensity in the mob. We name it the emotional circle effect. It means that, when the 


\begin{tabular}{|l|r|r|r|}
\hline & without emotion & with emotion but no propagation & with emotion and propagation \\
\hline SR & 7.05 & 14.08 & 45.03 \\
\hline ER & 0 & 5.25 & 19.81 \\
\hline
\end{tabular}

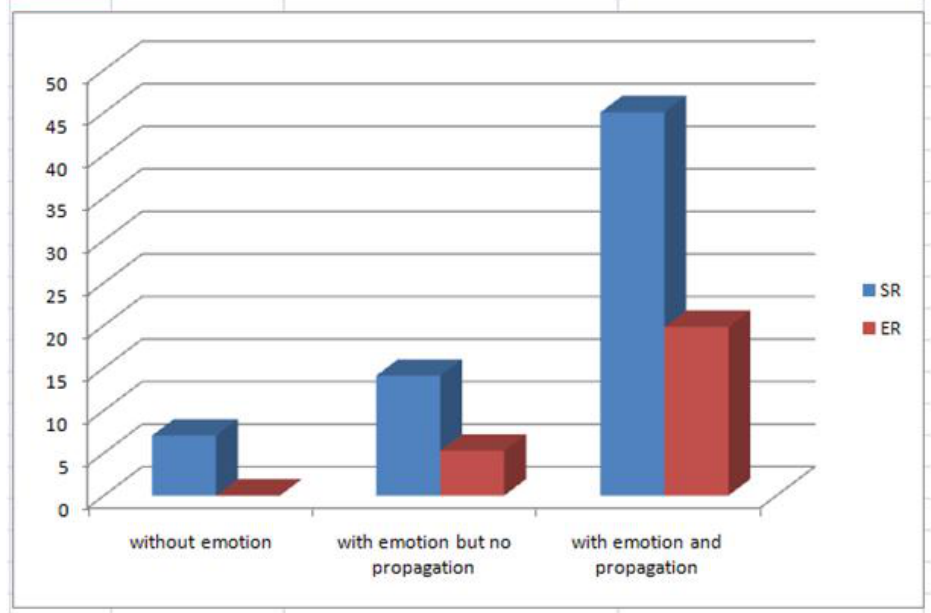

Fig. 10. Evolutions of the emotion rate and the number of survivors

first agent perceives the fire, he is scared and executes a behavior revealing his fear; the other agents perceive these behaviors; they are scared in turn and react to this emotion; the first agent mentioned perceives these behaviors and then the intensity of his own fear is increased. This type of process is repeated and the intensity of the fear in the mod jumps significantly. In a real situation, this kind of phenomenon happens frequently and is not limited to the fear inducing panic but also for example to the joy: the joy of the spectators in the stadium is much more intensive than that of the man who watches the match on his television at home.

\section{Conclusion}

Theoretically we proposed a model of emotion based on cognitive theories of emotion and then a architecture of emotional agent. According to the theory proposed, we can improve the emergency evacuation by adding emotional factors.

Practically we described the model of the simulation of the emergency evacuation in a burning shopping center. In addition, according to our simulation, we succeeded to prove the important role of emotions in the emergency evacuation.

In our research, we simulate only one emotion. The behaviors of agent are also affected by only one emotion. Simulating a multi-emotion model is thus the principal perspective of our research. In the multi-emotion model, each stimulus can trigger more than one emotion and, because many current emotions influence not only the appraisal process but also the behaviors; the simulation will thus become more and more complex. 
Acknowledgment. This work was funded by the project EPIS, a French IRD SPIRALES research program that is developed by research teamwork MSI-IRD UMMISCO.

\section{References}

1. Adam, C., Gaudou, B., Login, D., Lorini, E.: Logical modeling of emotions for ambient intelligence. In: Mastrogiovanni, F., Chong, N.Y. (eds.) Handbook of Research on Ambient Intelligence: Trends and Perspectives. IGI Global Publisher (2009) (to appear in 2010)

2. Amouroux, E., Chu, T., Boucher, A., Drogoul, A.: Gama: an environment for implementing and running spatially explicit multi-agent simulations. In: Pacific Rim International Workshop on Multi-Agents, Bangkoku, Thailand, pp. 359-371 (2007)

3. Arnold, M.B.: Emotion and personality. Columbia University Press, New York (1960)

4. Bosse, T., Duell, R., Memon, Z.A., Treur, J., van der Wal, C.N.: A Multi-Agent Model for Emotion Contagion Spirals Integrated Within a Supporting Ambient Agent Model. In: Yang, J.-J., Yokoo, M., Ito, T., Jin, Z., Scerri, P. (eds.) PRIMA 2009. LNCS (LNAI), vol. 5925, pp. 48-67. Springer, Heidelberg (2009)

5. Cabanac, M.: What is emotion? Behavioural Processes 60, 69-83 (2002)

6. Damasio, A.R.: Descartes' Error: Emotion, Reason, and the Human Brain. Putnam Pub. Group (1994)

7. De Rosis, F., Pelachaud, C., Poggi, I., Carofiglio, V., De Carolis, B.: From greta's mind to her face: Modelling the dynamics of affective states in a conversational embodied agent. International Journal of Human-Computer Studies 59, 81-118 (2003)

8. Forgas, J.: Mood and judgment: The affect infusion model (aim). Psychological Bulletin 117, 39-66 (1995)

9. Hollmann, C., Lawrence, P.J., Galea, E.R.: Introducing emotion modelling to agent-based pedestrian circulation simulation. In: PED 2010. NIST, Maryland (2010)

10. Lange, C., James, W.: The emotions. Hafner, New York (1967)

11. Lazarus, R.S.: Emotion and Adaptation. Oxford University Press (1991)

12. Musse, S.R., Thalmann, D.: Hierarchical model for real time simulation of virtual human crowds. IEEE Transactions on Visualization and Computer Graphics 7(2), 152-164 (2001)

13. Nair, R., Tambe, M., Marsella, S.: The role of emotions in multiagent team work. In: Fellous, J.M., Arbib, M. (eds.) Who Needs Emotions: the Brain Meets the Robot. Oxford University Press (2005)

14. North, M., Collier, N., Vos, J.: Experiences creating three implementations of the repast agent modeling toolkit. ACM Transactions on Modeling and Computer Simulation 16(1), 1-25 (2006)

15. Ortony, A., Clore, G., Collins, A.: The cognitive structure of emotions. Cambridge University Press, United Kingdom (1988)

16. Parunak, H.V.D., Bisson, R., Brueckner, S., Matthews, R., Sauter, J.: A model of emotions for situated agents. In: Proceedings of the Fifth International Joint Conference on Autonomous Agents and Multiagent Systems, AAMAS 2006, Hakodate, Hokkaido, Japan, pp. 993-995 (2006) 
17. Rao, A.S., Georgeff, M.P.: Modeling rational agents within a bdi-architecture. In: Allen, J.A., Fikes, R., Sandewall, E. (eds.) Proc. Second Int. Conf. on Principles of Knowledge Representation and Reasoning (KR 1991), pp. 473-484. Morgan Kaufmann Publishers (1991)

18. Ren, A., Chen, C., Luo, Y.: Simulation of Emergency Evacuation in Virtual Reality. Tsinghua Science Technology 13(5), 674-680 (2008)

19. Shendarkar, A., Vasudevan, K., Lee, S., Son, Y.J.: Crowd simulation for emergency response using BDI agents based on immersive virtual reality. Simulation Modelling Practice and Theory 16(9), 1415-1429 (2008)

20. Stroehle, J.: How do pedestrian crowds react when they are in an emergency situation - models and software Pedestrian behavior (2008)

21. Taillandier, P., Drogoul, A., Vo, D., Amouroux, E.: Gama: A Simulation Platform That Integrates Geographical Information Data, Agent-Based Modeling and Multi-Scale Control. In: Desai, N., Liu, A., Winikoff, M. (eds.) PRIMA 2010. LNCS(LNAI), vol. 7057, pp. 244-260. Springer, Heidelberg (2011)

22. Zoumpoulaki, A., Avradinis, N., Vosinakis, S.: A Multi-Agent Simulation Frame Work for Emergency Evacuations Incorporating Personality and Emotions. In: Konstantopoulos, S., Perantonis, S., Karkaletsis, V., Spyropoulos, C.D., Vouros, G. (eds.) SETN 2010. LNCS (LNAI), vol. 6040, pp. 423-428. Springer, Heidelberg (2010) 